\title{
Separación por sexo y caracteres productivos en un cruzamiento experimental de tres vías de pollo campero
}

\author{
Effect of separate sex growing on productive traits in a three-way \\ experimental cross of the free-range chicken
}

\author{
Antruejo Alejanda Edit ${ }^{1 *}$, Savoy Juan Pablo ${ }^{1}$, Montenegro Agustina ${ }^{2}$, \\ Savoy Julio Cesar ${ }^{1}$, Canet Zulma Edit ${ }^{2,4}$, Dottavio Ana María ${ }^{2,3}$, \\ Di Masso Ricardo JosÉ $\hat{\mathbf{2}}^{\mathbf{3}}$
}

1. Cátedra de Producción Avícola y Pilíferos. Facultad de Ciencias Veterinarias. Universidad Nacional de Rosario. 2. Cátedra de Genética. Facultad de Ciencias Veterinarias. Universidad Nacional de Rosario. 3. Instituto Nacional de Tecnología Agropecuaria, Estación Experimental Agropecuaria Pergamino "Ing. Agr. Walter Kugler" (INTA-EEA Pergamino). 4. Carrera del Investigador Científico de la Universidad Nacional de Rosario (CIC-UNR).

* Correo electrónico de la autora de contacto: aantruej@fveter.unr.edu.ar

\begin{abstract}
Resumen
El pollo Campero INTA es un ave con menor velocidad de crecimiento que los parrilleros comerciales cuya producción se encuentra protocolizada, si bien dicho protocolo no establece condiciones referidas a la modalidad de crianza mixta o con separación de sexos. El objetivo de este trabajo fue evaluar el efecto de dicha práctica sobre el patrón dinámico de crecimiento, la uniformidad por precisión, la conformación corporal y los caracteres a la faena en el cruzamiento experimental de tres vías Campero Casilda. Las aves se criaron en confinamiento como un único grupo hasta los 35 días y bajo dos modalidades (lotes mixtos y lotes de un único sexo) durante la etapa con acceso a parque, entre los 36 días y la faena a los 77 días de edad. La separación por sexo fue ventajosa para los machos, en tanto al crecer solos lo hicieron hacia un mayor peso asintótico y con menor tasa de maduración, lo que implica mayor eficiencia alimenticia. Este patrón no afectó el peso a la edad de faena ni el rendimiento y se tradujo en mayor proporción de los cortes de valor carnicero y menor proporción de grasa. Las hembras criadas en lotes por separado crecieron hacia un menor peso asintótico, con mayor tasa de maduración, relación desfavorable en términos de eficiencia alimenticia, alcanzaron menor peso a la edad de faena y, si bien, presentaron menor contenido de grasa e igual proporción de pata muslo, manifestaron una leve reducción en la proporción de pechuga y en el rendimiento.
\end{abstract}

\section{Palabras clave}

Crecimiento, uniformidad, conformación, caracteres a la faena

\begin{abstract}
Campero INTA is a type of bird with a slower growth rate than commercial broilers with a standardised production protocol; however, the protocol does not establish conditions related to sex separation during breeding. The aim of this work was to evaluate the effect of sex separation on body growth pattern, uniformity by precision, body conformation and slaughter traits in the three-way experimental cross Campero Casilda chickens. The birds were kept in confinement as a single group until 35 days of age and then separated into two treatments (mixed and single sex) during the free-range stage, between 36 days and slaughter at 77 days of age. Sex separation was advantageous for males which evidenced greater asymptotic weight and lower maturation rate, suggesting better feed efficiency. In addition, sex separation in males did not affect body weight at slaughter age or carcass yield and resulted in a greater proportion valuable muscle cuts and a lower proportion of body fat. On the other hand, females raised alone, evidenced lower asymptotic weight, and a higher maturation rate, suggesting lower feed efficiency. In addition, they reached a lower weight at slaughter age and, although they had a lower fat content and an equal proportion of thigh, showed a slight decrease in the proportion of breast and a reduction in yield.
\end{abstract}

\section{Key words}

Growth, uniformity, body conformation, slaughter traits

Fecha de recepción: 08/06/2018

Fecha de aprobación: 03/11/2018
ANALECTA VeT 2018; Julio-Diciembre; 38(2):10-17

Impresa ISSN 03655 14-8 Electrónica ISSN 1514-2590 


\section{Introducción}

La estrategia de mejoramiento genético para la denominada producción avícola orgánica, ecológica y/o de campo incluye opciones que van desde el diseño de programas independientes con producción de biotipos especiales hasta la integración de genotipos convencionales de la avicultura industrial a las nuevas propuestas de manejo (Dottavio \& Di Masso, 2010). El pollo Campero INTA es un ave con menor velocidad de crecimiento que los parrilleros comerciales, apto para su utilización en estos sistemas alternativos, cuya producción se encuentra protocolizada (Bonino, 1997). Dicho protocolo establece condiciones por cumplir para la certificación del producto obtenido, similares a las descritas para el pollo Label Rouge francés (Sauveur, 1997). Entre ellas, pueden mencionarse las densidades de aves permitidas y las edades mínimas y máximas de faena, sin referirse específicamente a la modalidad de crianza mixta o con separación de sexos. En los sistemas intensivos dedicados a la producción de pollos parrilleros se considera deseable que las aves expresen, con un elevado grado de ajuste, el patrón de crecimiento promedio propio de su estirpe. Es por ello que, si bien el manejo pretende maximizar la ganancia de peso, es esperable que este objetivo se logre con la menor dispersión posible, de manera tal que pueda asegurarse una alta uniformidad del lote (van de Ven, 2008). Si bien en los sistemas alternativos la uniformidad es menos trascendente que en la avicultura industrial, ya que la imagen del pollo de campo está asociada con una cierta variabilidad en el tamaño y, por ende, en el peso corporal, esta sigue siendo una característica deseable por su vinculación con aspectos de manejo. Uno de los factores que atenta contra la uniformidad de los lotes mixtos es el dimorfismo sexual evidente en las aves para carne, incluido el pollo campero (Librera et al., 2012) y observable en el Campero Casilda (Canet et al., 2017; Savoy et al., 2014), un tipo de ave que se faena en una edad que duplica la de los pollos parrilleros actuales. El objetivo de este trabajo fue evaluar el efecto de la separación por sexos versus la cría en lotes mixtos sobre el patrón dinámico de crecimiento, la uniformidad por precisión del peso corporal, la conformación corporal y los caracteres a la faena en un cruzamiento experimental de tres vías de pollo campero.

\section{Materiales y métodos}

Se evaluaron machos y hembras del híbrido experimental de tres vías Campero Casilda, producto del cruzamiento de gallos de la población sintética AH' (50 \% Hubbard 50 \% estirpe Anak grises) con hembras derivadas del cruzamiento simple entre gallos de la población sintética ES (87,5 \% Cornish Colorado, 12,5 \% Rhode Island Red) y gallinas de la población sintética A (75 \%
Cornish Colorado 25 \% Rhode Island Red). Las poblaciones mencionadas se generaron y mantienen en la Sección Aves de la Estación Experimental Agropecuaria (EEA) "Ing. Agr. Walter Kugler" del Instituto Nacional de Tecnología Agropecuaria (INTA), en Pergamino, donde se llevaron a cabo los cruzamientos y las incubaciones. En el momento del nacimiento, los pollitos se sexaron por cloaca, se identificaron con banda alar numerada y se vacunaron contra la enfermedad de Marek. Las aves del ensayo se criaron en las instalaciones de la Facultad de Ciencias Veterinarias de la Universidad Nacional de Rosario (UNR) de acuerdo con las especificaciones del protocolo para la producción de pollos camperos (Bonino, 1997) como un único grupo, en confinamiento, hasta los 35 días de edad y bajo dos modalidades (lotes mixtos y lotes de un único sexo; $\mathrm{n}=16$ aves de cada sexo por modalidad de crianza) durante la etapa con acceso a parque, entre los 36 días y la faena a los 77 días de edad.

\section{Patrón dinámico del peso corporal}

Se registró el peso corporal individual de todas las aves con intervalos semanales entre el nacimiento y la faena. Los datos longitudinales peso corporal-edad cronológica se ajustaron por regresión no lineal con la función sigmoidea de Gompertz (Fitzhugh, 1976):

$$
\mathrm{Wt}=\mathrm{A} \exp (-\mathrm{b} \exp (-\mathrm{kt}))
$$

donde: $\mathrm{Wt}=$ peso corporal $(\mathrm{g})$ en el tiempo $\mathrm{t}, \mathrm{A}=$ peso corporal maduro $\mathrm{o}$ asintótico $(\mathrm{g}), \mathrm{b}=$ parámetro de posición (constante de integración sin significado biológico), $\mathrm{k}=$ tasa de maduración o de aproximación al valor asintótico A (g-1) y t = edad en semanas. Para la evaluación de la bondad de los ajustes se utilizaron tres criterios: la convergencia de las iteraciones en una solución, el valor del coeficiente de determinación no lineal ajustado (R2) y la aleatoriedad en la distribución de los residuales determinada con un test de rachas o ciclos (Sheskin, 2011). A los efectos del análisis estadístico los valores individuales de los estimadores de los dos parámetros con significado biológico (A y k) se consideraron nuevas variables aleatorias.

\section{Uniformidad en peso corporal}

La uniformidad del peso corporal de cada grupo en los mismos intervalos de tiempo (11 valores) se evaluó a partir del cálculo del coeficiente de variación para peso corporal [desvío estándar fenotípico relativizado por el valor del promedio del carácter y expresado como porcentaje (Toudic, 2009)]. Los lotes se categorizaron en: muy uniformes $(\mathrm{CV}<8 \%)$, uniformes $(\mathrm{CV}=8$ $10 \%)$, de uniformidad moderada $(\mathrm{CV}=10-12 \%)$ y de uniformidad deficiente (CV > $12 \%$ ) tomando 
como base una escala utilizada por una compañía comercial (Aviagen, 2010). A partir de los 35 días de edad se trabajó con tres grupos experimentales: machos solos, hembras solas y lotes mixtos. Los grupos controlados a partir de los 35 días estuvieron conformados por 32 aves. Dado que el lote mixto contó con 16 machos y 16 hembras, para los efectos de los cálculos solo se consideraron 16 de las 32 aves totales controladas en los lotes de un único sexo.

\section{Conformación corporal prefaena}

El día previo a la faena se registraron las siguientes variables sobre el animal vivo (Latshaw \& Bishop, 2001):

-longitud de la caña,

-longitud de la tibia,

-longitud de la pechuga (distancia en $\mathrm{cm}$ entre el vértice de la quilla del esternón y el sitio de unión de las clavículas),

-ancho de la pechuga (ancho del tórax, en cm, a la altura de la unión de las clavículas, según Mallo et al., - 1999),

-longitud dorsal (longitud en cm medida con cinta métrica y con el ave de pie, desde el nadir de la curva que forma el cuello con el cuerpo hasta la base de la cola),

-longitud dorsal parcial (porción de la longitud anterior tomada en su inicio a la altura del húmero y en su porción final a la altura del fémur),

-distancia interhúmeros,

-distancia interfémures,

-circunferencia corporal (longitud, en $\mathrm{cm}$, a la altura de la porción anterior del borde del esternón, pasando por debajo de las alas y por delante de las patas).

Sobre la base de dichas mediciones, para cada ave se calcularon cuatro índices de conformación (Dottavio et al., 2010): índice 1 (longitud de la tibia/longitud de la caña), índice 2 (longitud de la pechuga/ancho de la pechuga, indicador de la forma de dicho corte), índice 3 (ancho entre húmeros/ancho entre fémures, indicador de la conformación del ave vista desde dorsal) e índice 4 (circunferencia corporal/longitud dorsal, indicador de la conformación volumétrica del ave).

\section{Caracteres en la faena}

A la faena se registró, en 16 aves de cada sexo por modalidad de crianza, el peso corporal PC (g), el peso eviscerado PE (g), el peso (g) de dos cortes valiosos (pechuga con hueso y pata muslo derecho) y el peso (g) de la grasa abdominal. El porcentaje de cada corte (pechuga: PP y pata muslo: PM) y de la grasa abdominal (G) se calculó como proporción del peso eviscerado. El rendi- miento se calculó como [(peso eviscerado/peso pre-faena) $\mathrm{x}$ 100)].

\section{Análisis estadístico}

El efecto del sexo, del tipo de crianza y de la interacción entre ambos factores principales sobre los estimadores de los parámetros de la función de Gompertz con significado biológico (A y k) y los índices de conformación corporal, el peso eviscerado, las proporciones de pechuga, de patamuslo y de grasa y sobre el rendimiento a la faena se evaluaron con un análisis de la variancia correspondiente a un diseño completamente aleatorizado con un experimento factorial 2x2 (dos sexos $\mathrm{x}$ dos modalidades de crianza).

\section{Resultados}

\section{Patrón dinámico del peso corporal}

La Tabla 1 resume el comportamiento de los estimadores de la función de Gompertz con significado biológico. Se observaron efectos significativos de la interacción entre sexo y modalidad de crianza sobre el peso corporal asintótico $(F=16,0$; $\mathrm{P}=0,0002)$ y sobre la tasa de maduración para el peso corporal $(\mathrm{F}=17,5 ; \mathrm{P}<0,0001)$. Esta observación relativiza la interpretación de los resultados sobre los efectos principales, si bien la modalidad de crianza no afectó en forma significativa el comportamiento de ninguno de los dos paráme$\operatorname{tros}(\mathrm{A}: \mathrm{F}=0,58 ; \mathrm{P}=0,448 ; \mathrm{k}: \mathrm{F}=3,0 ; \mathrm{P}=$ o,o88) y el sexo afectó a ambos de acuerdo con lo esperado: mayor peso corporal asintótico en machos (machos: $4820 \mathrm{~g}$ vs. hembras: $3346 \mathrm{~g}-\mathrm{F}=$ 173; $\mathrm{P}=0$,0002) y mayor tasa de maduración promedio para peso corporal en hembras (machos: 0,0308 vs. hembras: 0,$0331 ; \mathrm{F}=8,52 ; \mathrm{P}=0,005$ ). En la figura 1 se observan las trayectorias de las curvas de crecimiento de machos y hembras bajo las dos modalidades de crianza.

Tabla 1. Estimadores de los parámetros de la función de Gompertz de machos y hembras Campero Casilda criados en lotes mixtos y con separación de sexos.

\begin{tabular}{lcccc}
\hline & \multicolumn{2}{c}{ Machos } & \multicolumn{2}{c}{ Hembras } \\
\cline { 2 - 5 } & $\begin{array}{c}\text { Cría } \\
\text { mixta }\end{array}$ & Sexos separados & $\begin{array}{c}\text { Cría } \\
\text { mixta }\end{array}$ & $\begin{array}{c}\text { Sexos } \\
\text { separados }\end{array}$ \\
\hline $\begin{array}{l}\text { A: Peso corporal } \\
\text { asintótico }(\mathrm{g})\end{array}$ & $4639 \pm 145$ & $5001 \pm 110$ & $3612 \pm 106$ & $3079 \pm 74$ \\
$\begin{array}{l}\text { k: Tasa de } \\
\text { maduración }\left(\mathrm{g}^{-1}\right)\end{array}$ & $0,0317 \pm 0,00075$ & $0,0299 \pm 0,00063$ & $0,0308 \pm 0,00085$ & $0,0353 \pm 0,0008$ \\
\hline
\end{tabular}

Todos los valores corresponden a la media aritmética \pm Error Estándar. Tamaño muestral: $\mathrm{n}=16$ aves por grupo experimental. 

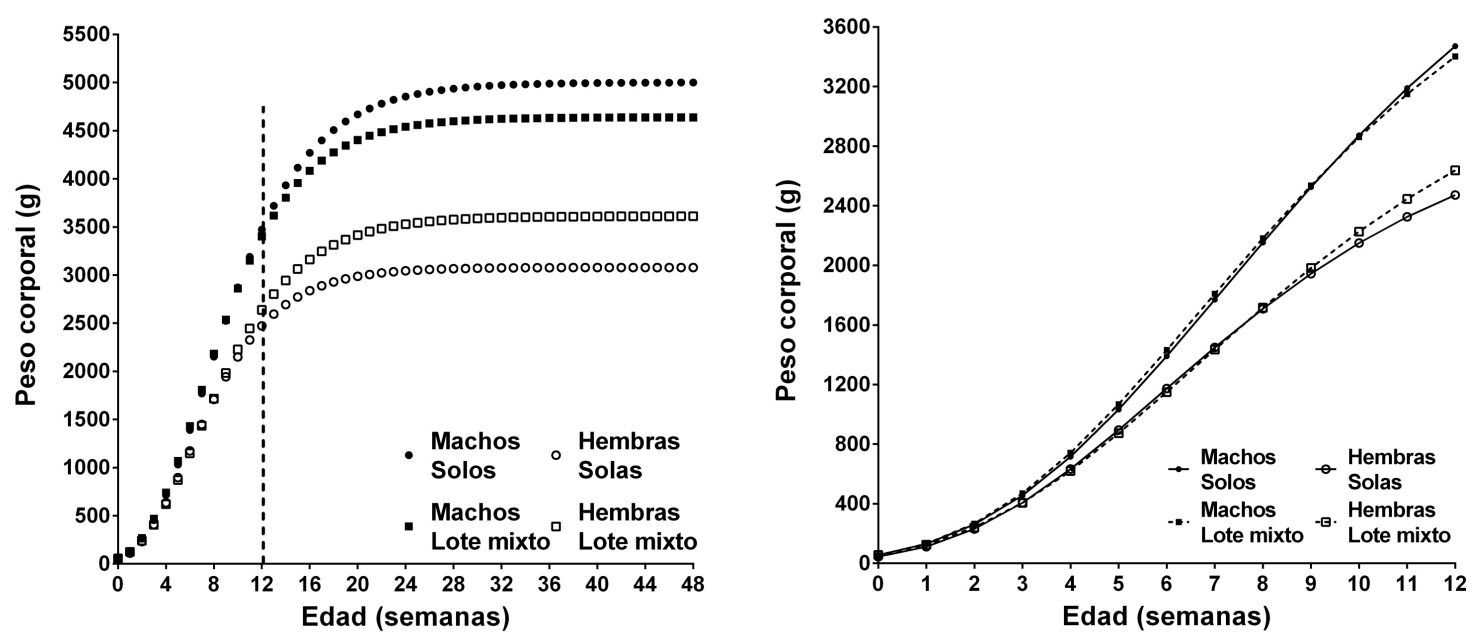

Figura 1. Curvas de crecimiento de aves machos y hembras Campero Casilda bajo dos modalidades de crianza durante las etapas de recría y terminación (Izquierda: crecimiento teórico hasta la madurez. Derecha: crecimiento durante el ciclo de producción).

\section{Uniformidad en peso corporal}

En la figura 2 se observa el comportamiento del indicador de uniformidad por precisión para peso corporal en las aves de cada sexo bajo las dos modalidades de cría durante las etapas de recría y terminación. De acuerdo con el criterio mencionado, el lote mixto mostró, a lo largo de todo el ciclo, una uniformidad deficiente (CV > $12 \%)$ explicable en términos del dimorfismo sexual propio de la especie. Cuando, a partir de los 35 días de edad, coincidentemente con el pasaje de la cría en confinamiento a la cría con acceso a parque, las aves se separaron en lotes por sexo, se constató una mejora sustancial en los valores del indicador, mostrando, tanto el lote de machos como el lote de hembras, valores del CV inferiores al $8 \%$ (lotes muy uniformes), particularmente en el caso de los machos con valores iguales o menores al $6 \%$. Las aves criadas en lotes mixtos durante esta etapa mantuvieron bajos niveles de uniformidad con valores crecientes del coeficiente de variación del peso corporal.

\section{Conformación corporal prefaena}

La Tabla 2 resume los valores de los índices de conformación evaluados en el día previo a la faena. El efecto de la interacción entre el sexo y la modalidad de crianza solo fue significativo en el caso del índice $1(\mathrm{~F}=6,33 ; \mathrm{P}=0,015)$, atribuible a un aumento del mismo en las hembras criadas en lotes mixtos, efecto no evidente en los machos. Independientemente del manejo, los machos presentaron mayor valor promedio del índice 2 que las hembras (2,140 vs. 1,910; $\mathrm{F}=23,5 ; \mathrm{P}<0,0001) \mathrm{e}$ independientemente del sexo, las aves en lotes mixtos presentaron menor valor promedio del índice 2 (2,048 vs. 2,002; F = 16,0; P = 0,0002) y a mayor valor del índice correspondieron pechugas más alargadas. No se observó efecto significativo del sexo sobre el valor promedio del índice $3(\mathrm{~F}=$ $0,011 ; \mathrm{P}=0,931)$ que en todos los casos fue menor a 1 , indicando aves que tienden a ensancharse hacia caudal. Independientemente del sexo, las aves criadas en lotes mixtos presentaron menores valores promedio del índice $(0,914$ vs. 0,$931 ; \mathrm{F}=$ $4,65 ; \mathrm{P}=0,0352)$ con cuerpos más angostos hacia craneal. La modalidad de crianza no afectó en forma significativa $(\mathrm{F}=0,021 ; \mathrm{P}=0,878)$ los valores del índice 4 . Independientemente del manejo de la alimentación las hembras presentaron cuerpos más voluminosos y los machos cuerpos más estilizados (1,390 vs. 1,$268 ; \mathrm{F}=38,3 ; \mathrm{P}<$ 0,0001).

Tabla 2. Índices de conformación a la edad de faena, de machos y hembras Campero Casilda criados en lotes mixtos y con separación de sexos.

\begin{tabular}{lcccc}
\hline & \multicolumn{2}{c}{ Machos } & \multicolumn{2}{c}{ Hembras } \\
\cline { 2 - 5 } & Cría mixta & Sexos separados & Cría mixta & Sexos separados \\
\hline Índice 1 & $1,429 \pm 0,0223$ & $1,431 \pm 0,0148$ & $1,586 \pm 0,0293$ & $1,486 \pm 0,0083$ \\
Índice 2 & $2,211 \pm 0,0549$ & $2,068 \pm 0,0591$ & $1,792 \pm 0,0181$ & $2,028 \pm 0,0461$ \\
Índice 3 & $0,916 \pm 0,0070$ & $0,928 \pm 0,0097$ & $0,912 \pm 0,0090$ & $0,934 \pm 0,0058$ \\
Índice 4 & $1,274 \pm 0,0174$ & $1,261 \pm 0,0151$ & $1,384 \pm 0,0202$ & $1,395 \pm 0,0238$ \\
\hline
\end{tabular}

Todos los valores corresponden a la media aritmética \pm Error Estándar. Tamaño muestral: $\mathrm{n}=16$ aves por grupo experimental.

\section{Caracteres productivos a la faena}

Los valores correspondientes a los caracteres productivos evaluados en el momento de la faena se presentan en la Tabla 3. Se observó un efecto no significativo de la interacción $(\mathrm{F}=$ 1,62; $\mathrm{P}=0,208)$ y efectos significativos del sexo $(\mathrm{F}=239 ; \mathrm{P}<0,0001)$ y del manejo $(\mathrm{F}=3,88 ; \mathrm{P}=$ 


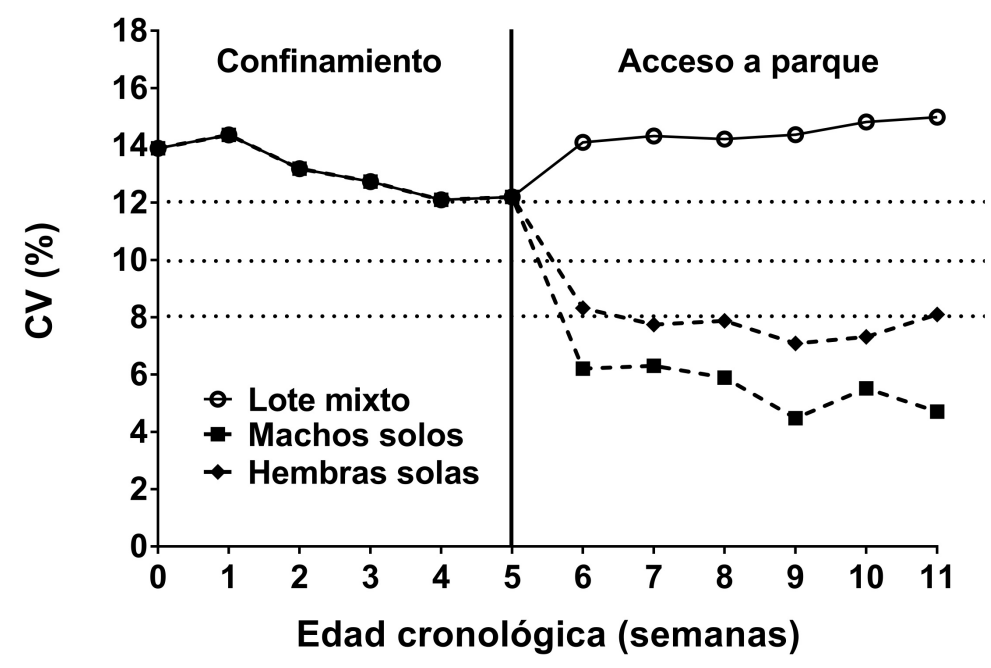

Figura 2. Uniformidad por precisión para peso corporal en aves machos y hembras Campero Casilda bajo dos modalidades de crianza durante las etapas de recría y terminación.

o,054) sobre el peso corporal prefaena y el peso eviscerado: los mayores valores promedio correspondieron a los machos y a las aves criadas en lote mixto. La interacción (sexo x manejo) fue estadísticamente significativa en el caso de la proporción de pechuga $(\mathrm{F}=4,89 ; \mathrm{P}=0,031) \mathrm{y}$, en menor medida, sobre el peso de pata muslo $(\mathrm{F}=3,54 ; \mathrm{P}=$ 0,065 ) atribuible a que la modalidad de crianza sólo afectó el valor promedio del carácter en machos. Con respecto a la proporción de grasa abdominal, ningún efecto resultó estadísticamente significativo (sexo: $\mathrm{F}=0,51 ; \mathrm{P}=0,480$; manejo: $\mathrm{F}=1,13 ; \mathrm{P}=0,292 ;$ interacción: $\mathrm{F}=0,01 ; \mathrm{P}=$ 0,991). No se observó interacción (sexo x manejo) sobre el rendimiento a la faena $(\mathrm{F}=2,08 ; \mathrm{P}=$ $0,154)$. El valor promedio de este carácter no se vio afectado por la modalidad de crianza $(\mathrm{F}=2,00$; $\mathrm{P}=0,162)$ pero sí por el sexo $(\mathrm{F}=5,13 ; \mathrm{P}=0,027)$, de manera que el mayor rendimiento a la faena correspondió a las hembras $(72,6 \%$ vs. $71,8 \%)$

Tabla 3. Caracteres a la faena de machos y hembras Campero Casilda criados en lotes mixtos y con separación de sexos.

\begin{tabular}{lcccc}
\hline & \multicolumn{2}{c}{ Machos } & \multicolumn{2}{c}{ Hembras } \\
\cline { 2 - 5 } & Cría mixta & Sexos separados & Cría mixta & Sexos separados \\
\hline $\begin{array}{l}\text { Peso corporal } \\
\text { prefaena (g) }\end{array}$ & $3204 \pm 51$ & $3186 \pm 37$ & $2475 \pm 52$ & $2339 \pm 45$ \\
$\begin{array}{l}\text { Peso corporal } \\
\text { eviscerado (g) }\end{array}$ & $2300 \pm 39$ & $2287 \pm 25$ & $1811 \pm 44$ & $1685 \pm 30$ \\
$\begin{array}{l}\text { Proporción de } \\
\text { pechuga (\%) }\end{array}$ & $24,6 \pm 0,36$ & $26,4 \pm 0,33$ & $27,5 \pm 0,59$ & $27,0 \pm 0,62$ \\
$\begin{array}{l}\text { Proporción de } \\
\text { pata-muslo (\%) }\end{array}$ & $15,5 \pm 0,34$ & $16,3 \pm 0,20$ & $14,5 \pm 0,12$ & $14,5 \pm 0,13$ \\
$\begin{array}{l}\text { Proporción de } \\
\text { grasa abdominal } \\
\text { (\%) }\end{array}$ & $2,39 \pm 0,184$ & $2,13 \pm 0,178$ & $2,22 \pm 0,328$ & $1,96 \pm 0,247$ \\
$\begin{array}{l}\text { Rendimiento a } \\
\text { la faena (\%) }\end{array}$ & $71,8 \pm 0,37$ & $71,8 \pm 0,22$ & $73,1 \pm 0,49$ & $72,1 \pm 0,26$ \\
\hline
\end{tabular}

Todos los valores corresponden a la media aritmética \pm Error Estándar. Tamaño muestral: $\mathrm{n}=16$ aves por grupo experimental.

\section{Discusión}

El pollo Campero INTA es un tipo de ave para carne producido a partir de cruzamientos entre poblaciones sintéticas propias del núcleo genético de la Sección Aves en la EEA de INTA en Pergamino. El protocolo para su producción (Bonino, 1997) no incluye restricciones, ni tampoco recomendaciones, acerca de la conveniencia o no de criar a las aves de ambos sexos como un único lote o, por el contrario, hacerlo en lotes por separado.

El pollo Campero Casilda es un cruzamiento experimental, en evaluación como potencial genotipo doble propósito destinado para propuestas de avicultura alternativa con respecto al modelo intensivo. En este contexto, se requiere de información que posibilite una toma de decisiones fundadas con respecto a las prácticas de manejo. Las hembras que se utilizan como ponedoras deben criarse separadas de los machos, ya que por su condición de aves pesadas deben ser restringidas en el aporte de nutrientes, mientras que si su destino es la producción de carne se las podría criar conjuntamente con ellos, con alimentación a voluntad. Los principales efectos de la práctica de cría de machos y hembras en conjunto o por separado se relacionan con potenciales modificaciones en el patrón global de crecimiento (peso corporal, uniformidad y conformación) y en el rendimiento, total y por cortes, en la faena. El efecto de la interacción entre sexo y modalidad de crianza sobre los estimadores de los parámetros de la función de Gompertz puso de manifiesto la diferente respuesta de machos y hembras ante el cambio de manejo. La cría en lotes mixtos afectó en forma negativa el peso corporal asintótico estimado en el caso de los machos y en forma positiva en el caso de las hembras. En consecuencia, el dimorfismo sexual en peso resultó ser mayor 
en las aves criadas en lotes de sexo separado (peso de las hembras $=62 \%$ del peso de los machos) que en aves criadas en lotes mixtos (peso de las hembras $=78 \%$ del peso de los machos). El efecto de la interacción sobre la tasa de maduración, por su parte, se explica porque los machos criados en lotes mixtos presentaron mayor tasa de maduración que aquellos criados en lotes separados por sexo, mientras que en las hembras se observó la respuesta inversa (menor tasa de maduración en lotes mixtos). Como resultado de estos comportamientos, las hembras presentaron mayor tasa de maduración que los machos criados por separado mientras que dicha diferencia se hizo no significativa cuando las aves se criaron en lotes mixtos. Por lo tanto, en lo que al patrón de crecimiento se refiere, este fue más favorable (mayor peso asintótico y menor tasa de maduración) para los machos cuando se los crió solos. En el caso de las hembras, si bien cuando se las crió en lotes mixtos crecieron hacia un mayor peso asintótico lo hicieron con menor velocidad, lo que redujo el efecto final sobre el peso corporal desde un $17 \%$ en la asíntota hasta un 5,8 \% en la edad de sacrificio. Esta evidencia indica la conveniencia de separar a las aves por sexo, separación que podría llevarse a cabo al trasladarlas a los alojamientos con acceso a parque. En ese momento la expresión de caracteres sexuales secundarios posibilita llevar a cabo la selección obviando la necesidad del sexado por inspección de la cloaca en el nacimiento, que requiere de personal adiestrado y supone un aumento en el costo del pollito BB. Esta separación, como es evidente dado el dimorfismo sexual de la especie, se traduce en una mayor uniformidad de los lotes. Las aves de uno y otro sexo criadas en lotes mixtos tienden a mostrar una reducción en las medidas lineales que definen su conformación, por lo que la modalidad de manejo afectó la conformación corporal de las aves evaluada a partir del cálculo de cuatro índices. Pese a los significados estadísticos observados en los índices de conformación, la trascendencia biológica de tales modificaciones queda minimizada ante lo exiguo de las diferencias observadas y, en tal sentido, la importancia productiva de tales modificaciones está vinculada con un potencial impacto sobre los cortes de interés carnicero y el rendimiento en la faena. $\mathrm{Al}$ respecto, la cría de las aves de uno y otro sexo por separado no afecta ni el rendimiento ni la proporción de grasa en la faena. Independientemente del sexo, la cría por separado afecta negativamente el valor del peso corporal prefaena y del peso eviscerado con una reducción de mayor magnitud en el caso de las hembras (5,8 \% y 7,5 \%, respectivamente) que en los machos (o,6\%). La presencia de interacciones significativas determina una respuesta favorable sobre la proporción de pechuga en las hembras (aumento) y desfavorable en el caso de los machos (disminución) tanto sobre este corte como sobre la proporción de pata muslo. Sam et al. (2010), trabajando con pollos parrilleros, no encontraron evidencia de que la separación por sexos presentara ventajas cuando el ciclo productivo finalizaba a las 8 semanas de edad. Por el contrario, cuando dicho ciclo se extendía más allá de la edad mencionada la práctica de criar a los machos separados de las hembras se presentaba como ventajosa debido a un mejor desempeño de los machos. En coincidencia con esta última observación, y tomando en consideración la duración del ciclo productivo del pollo campero, se concluye que la práctica de separar a las aves Campero Casilda por sexo es ventajosa para los machos. Al crecer solos, lo hacen hacia un mayor peso asintótico y con menor tasa de maduración, lo que implica una mayor eficiencia alimenticia, patrón que, sin afectar el peso a la edad de faena ni el rendimiento, se traduce en una mayor proporción de los cortes de valor carnicero y menor proporción de grasa. Este comportamiento es coincidente con el informado por Laseinde y Oluyemi (1994) quienes, si bien concluyen que la diferencia no se traduce en ventajas significativas de la práctica, observaron mayor superioridad porcentual de los machos cuando se los cría separados de las hembras. Por su parte, las hembras criadas en lotes por separado crecen hacia un menor peso asintótico y con mayor tasa de maduración, relación desfavorable en términos de eficiencia alimenticia, alcanzan un peso menor a la edad de faena $y$, si bien, presentan menor contenido de grasa y la misma proporción de pata muslo, presentan un leve deterioro en la proporción de pechuga y una reducción en el rendimiento. La mayor relación de conversión de las aves criadas en sistemas semiextensivos representa un aspecto determinante para la adopción de dicha modalidad productiva por parte de aquellos interesados en aprovechar un nicho del mercado que demanda productos generados sobre la base del respeto por el bienestar animal. El alimento representa el mayor componente de los costos y ello torna imprescindible contar con un mayor precio de venta para asegurar la rentabilidad del emprendimiento. En este sentido, el sexo de las aves de crecimiento lento juega un papel de mayor impacto que su genotipo. Dottavio et al. (2013) estimaron la eficiencia alimenticia de machos y hembras de los híbridos experimentales de tres vías Campero Casilda y Campero Pergamino en comparación con el cruzamiento simple Campero INTA como genotipo de referencia, entre los 42 y los 77 días de edad. Independientemente del sexo, no observaron diferencias significativas en la eficiencia ni en sus variables asociadas (ganancia de peso y consumo acumulado de alimento) entre genotipos pero, en los tres grupos genéticos, los machos fueron más pesados y si bien tuvieron mayor consumo medio diario también presentaron mayor aumento medio diario de peso, por lo que mostraron mayor eficiencia alimenticia y menor relación de conversión que las hembras.

En términos generales, la evidencia empírica indica que la separación por sexos sería la modalidad de cría más recomendada. Si el pollo 
Campero Casilda se utiliza como genotipo doble propósito, las hembras deben ser restringidas a partir de la $5^{\text {a }}$ semana de vida para asegurar un correcto desempeño reproductivo (Barbato, 1999). Bajo este esquema los sexos deben ser criados, necesariamente, por separado. $\mathrm{Si}$, por el contario, todas las aves se destinan a producción de carne, la cría con separación de sexos favorece a los machos, mientras que la cría en lotes mixtos favorece a las hembras. En caso de ser este el destino de las aves, y dado que el protocolo de producción de pollos Campero INTA (Bonino, 1997) establece un rango de edades de sacrificio entre 75 y 90 días, en el caso de trabajar con sexos separados, las hembras pueden ser faenadas a mayor edad que los machos si la ventaja de prolongar el ciclo en términos de peso a la faena compensa la pérdida esperable en la eficiencia de producción de esa ganancia de peso.

\section{Agradecimientos}

Se agradece la responsable colaboración de los alumnos de la Facultad de Ciencias Veterinarias de la Universidad Nacional de Rosario, cuyo trabajo contribuyó con la concreción de este Proyecto, y al INTA Pergamino, en cuyas instalaciones se llevó a cabo la faena de las aves.

\section{Conflicto de intereses}

No existe conflicto de intereses, incluyendo entre estos últimos las relaciones financieras, personales o de otro tipo con otras personas $\mathrm{u}$ organizaciones que pudieran influir de manera inapropiada en el trabajo.

\section{Bibliografía}

Aviagen. Manual de manejo del pollo de carne Ross, pág.72. 2010. Disponible en: http:// es.aviagen.com/assets/Tech_Center/

BB_Foreign_Language_Docs/Spanish_TechDocs/ Manual-del-pollo-Ross.pdf. $\quad$ [Consultado 24/09/2018].

Barbato GF. 1999. Genetic relationships between selection for growth and reproductive effectiveness. Poulty Science 78(3):444-52.

doi: $10.1093 / \mathrm{ps} / 78.3 .444$

Bonino MF. 1997. Pollo Campero. Protocolo para la certificación. INTA. EEA Pergamino.

Canet ZE, Savoy JP, Antruejo AE, Martines A, Romera BM, Dottavio AM, Di Masso RJ. Dimorfismo sexual para crecimiento dimensional en el híbrido experimental de tres vías Campero Casilda. XIX Congreso y XXXVII Reunión Anual de la Sociedad de Biología de Rosario. 2017. Disponible en: https://www.sbr.org.ar/Libros/ libro_resumenes_2017.pdf. 24/09/2018].

Castelló Llobet JA, Pontes Pontes M, Franco González F. 1989. Producción de huevos. Real Escuela de Avicultura, Barcelona, España. pp. 85.

Cobb Breeder Management Guide. 1998. CobbVantress, Inc., Siloam Spring, AR.

Dottavio AM, Amoroto I, Romera BM, Álvarez M, Canet ZE, Di Masso RJ. 2010. Conformación corporal en poblaciones de pollos para carne con diferente velocidad de crecimiento. Revista FAVE, Sección Ciencias Veterinarias, 9(2):25-36.

doi: 10.14409/favecv.v9i2.1500

Dottavio AM, Di Masso RJ. 2010. Mejoramiento avícola para sistemas productivos semi-intensivos que preservan el bienestar animal. BAG, Journal of Basic and Applied Genetics. 21(2).Art. 12:1-10.

Hudson BP, Lien RJ, Hess JB. 2001. Effects of body weight uniformity and pre-peak feeding programs on broiler breeder hen performance. Journal of Applied Poultry Research. 10:24-32.

doi: 10.1093/japr/10.1.24

Laseinde EAO, Oluyemi JA. 1994. Effect of sex separation at the finisher phase on the comparative growth performance, carcass characteristics and breast muscle development between male and female broiler chicken. Nigerian Journal of Animal Production. 21:11-18.

Latshaw JD, Bishop BL. 2001. Estimating body weight and body composition of chickens by using noninvasive measurements. Poultry Science 80:868-73. doi: 10.1093/ps/80.7.868

Librera JE, Álvarez M, Lucach S, Serrano C, Velázquez J, Canet Z E, Dottavio AM, Di Masso RJ. 2012. Comportamiento del dimorfismo sexual en peso corporal en función de la edad en híbridos experimentales de pollos camperos. XIV Congreso y XXXII Reunión Anual de la Sociedad de Biología de Rosario. pp 272. Disponible en: http:// www.sbr.org.ar/Libros/libro_resumenes_2012.pdf. [Consultado 23/09/2018].

Mallo G, Villar E, Melo J, Miquel MC, Capelletti C, Paolella M. 1999. Correlaciones fenotípicas y ecuaciones de regresión para estimaciones del peso y proporción de la pechuga. XVI Congreso Latinoamericano de Avicultura. 435-39.

Sam IM, Akpa GN, Alphonsus C, IyegheErakpotobor GI, Agubosi OCP. 2010. Effect of sex separation on growth performance and carcass characteristics of broilers raised to maturity. Continental Journal of Animal And Veterinary 
Research 2:35-40.

doi: 10.5281/zenodo.818029

Sauveur B. 1997. Les critères et facteurs de la qualité des poulets Label Rouge. INRA Production Animal 10: 219-26.

Savoy JP, Canet ZE, Dottavio AM, Antruejo AE, Di Masso RJ. 2014. Dimorfismo sexual para peso corporal temprano y tasa de crecimiento exponencial pre-inflexión en pollos Campero Casilda. VIII Jornada de Ciencia y Tecnología de la Universidad Nacional de Rosario.

Sheskin DJ. 2011. Handbook of parametric and nonparametric statistical procedures. Chapman \& Hall, USA.

Terraes JC, Sindik M, Revidatti F, Rigonatto T, Sandoval GL, Simón J. 2008. Efectos de la composición de la ración sobre el porcentaje de uniformidad en pollos parrilleros. XXVIX Sesión de Comunicaciones Científicas. Facultad de Ciencias Veterinarias. UNNE.
Terraes JC, Sindik M, Sandoval L, Revidatti F, Fernández R, Simón J. 2007. Efectos de dos dietas de diferente composición sobre el porcentaje de uniformidad de pollos parrilleros hembras. XXVIII Sesión de Comunicaciones Científicas. Facultad de Ciencias Veterinarias. UNNE.

Toudic C. Evaluating uniformity in broilers. Factors affecting variation. Disponible en https:// wenku.baidu.com/view/ aef8do34eefdc8d376ee32db.html [Consultado 24/09/2018].

van de Ven L. 2008. Maximizando la uniformidad del lote con técnicas de alto nivel. WorldPoultry.net. 26(7):10-13.

Veerapen DS, Driver BMF. 1999. Separate sex growing of Ross 208 broilers and effects on broiler performance and carcass quality. Science and Technology (4):145-59. 